\title{
Measurement of Deformation and Force for Microcantilevers Based on Feature Point Matching and Digital Image Correlation
}

\author{
Hongtao Liu, Zhenning Liang, Wen Hu, Jinqiu Mo and Shigang Wang* \\ School of Mechanical Engineering, Shanghai Jiao Tong University, \\ 800 Dongchuan Road, Shanghai, China
}

(Received April 9, 2014; accepted January 26, 2015)

Key words: microcantilever, micro force measurement, feature point matching, affine invariant feature descriptor, digital image correlation

The measurement of deformation and force for microcantilevers is an important issue in microassembly. An optical method based on local feature point matching and the digital image correlation (DIC) for deformation and force measurement of microcantilevers is proposed. Images of a microcantilever before and after deformation are obtained by an optical microscope. Using the Laplace-of-Gaussian (LOG) operator, feature points with local extreme response values are detected in the scale space in images before and after deformation. For each LOG feature point, an affine-invariant closed region is extracted based on which the sub-pixel position of the LOG point is relocated as the center of gravity of the closed region and an affine-invariant feature descriptor is constructed. Corresponding points in images before and after deformation are matched according to their affine invariant descriptors. To obtain the displacement of feature points with a higher resolution in bending deformation, the digital image correlation algorithm with a third-order transformation function is used and initialized by the sub-pixel information of matched points. The deformation of the microcantilever can be calculated by fitting the displacement of the matching points with the beam's bending deflection formula under the plane stress condition. Then, the force values as well as the position where the loader comes in contact with the cantilever are calculated through the least-squares method. The validity of the proposed method is verified through experiments on actual microcantilever deformation and comparison with other methods.

\section{Introduction}

With the development of micro-electromechanical systems (MEMS), microscale force measurement is becoming an important and actively researched area. As force loading and sensing are prerequisites for microassembly and mechanical behavior testing of microstructures, various methods and experimental setups have been developed *Corresponding author: e-mail: wangshigang@sjtu.edu.cn or liuhongtaosdu@163.com 
and applied for the measurement of force and deformation. ${ }^{(1,2)}$ Microcantilevers are important microcomponents that have various applications, such as actuators ${ }^{(3,4)}$ for micromanipulation and force sensors. ${ }^{(5-7)}$ The key component of the widely used atomic force microscope $(\mathrm{AFM})^{(8,9)}$ is the probe, which is a specific kind of cantilever. The calibration of the mechanical properties of microcantilevers is being much researched in the field of MEMS. ${ }^{(10-12)}$

There are several force sensing methods for microcantilevers, such as piezoelectric, ${ }^{(5)}$ piezoresistive, ${ }^{(6)}$ capacitive, ${ }^{(7)}$ and optical methods. ${ }^{(13-19)}$ Optical techniques are highly promising owing to their electromagnetic immunity and high resolution. In optical techniques, the deformation of microcantilevers is derived from images captured before and after the loading of external forces. Then, the force value can be obtained with precalibrated material properties, such as elastic modulus and Poisson's ratio. Zhou et al. ${ }^{(13)}$ proposed a method integrating vision feedback from an optical microscope and force feedback from an optical beam to perform microassembly with high speed and resolution, but there are difficulties caused by the alignment of the laser optics with respect to the elastic cantilever. Greminger and Nelson ${ }^{(14)}$ used a template matching method to determine the contour displacement field of the deformed object and recovered applied forces using Dirichlet-to-Neumann mapping. However, this method requires the location of the nearest point in the deformed image for each template vertex. Anis et al. ${ }^{(15)}$ proposed a template matching technique with the normalized cross-correlation method to determine the microgripper deflection and converted the deflection to force information using the relationship between tip force and displacement established by the finite element method (FEM). The specialized templates used in this technique were selected manually. Amiot et al.(16) utilized the optical phase field measured by an interferometric imaging setup to compute cantilever deflection from which applied forces and material properties of elastic modulus could be obtained. Li et al. ${ }^{(17)}$ used electronic speckle pattern interferometry (ESPI) to measure the deflection of a spring beam for a force sensor of the tensile test. The inteferometric techniques ${ }^{(16,17)}$ require complex equipment, which is expensive and sophisticated to implement. Hu and coworkers ${ }^{(18,19)}$ reduced the force measurement problem to that of pattern recognition using a support vector machine (SVM) to classify the feature vector of moment invariants and to predict the applied force. A large number of training samples need to be established in order to obtain accurate results for this method.

All of the above methods are based on the defection formula derived from EulerBernoulli theory. To detect the deformation of normal microsamples, markers ${ }^{(20-24)}$ are widely used to calculate the displacement information. Markers may be dark paints that are sprayed to the microspecimen, ${ }^{(20,21)}$ or array spots ${ }^{(22)}$ of different shapes obtained by milling at the end of a microcantilever, or microarrays ${ }^{(23)}$ indented into the surface of a thin film, or even gold lines ${ }^{(24)}$ deposited on silicon specimens for strain measurement. Besides the high cost for fabricating local features with high precision on the specimens, there are two additional issues that arise in the utilization of the manually specialized "marker" method. First, it may be destructive as it could cause changes in material properties, and second, algorithms for displacement measurement must be selected carefully to adapt to different markers. To obtain subpixel precision, the digital image 
correlation (DIC) method has been widely researched and applied. ${ }^{25-27)}$ DIC is usually used as a noncontact and full-field method to obtain the strain and crack information, ${ }^{(28)}$ or to determine the material properties of test samples such as elastic modulus and Poisson's ratio. ${ }^{(29)}$ DIC combined with the marker method can precisely calculate the displacement and strain. ${ }^{(22,23)}$

Based on the state-of-the-art force measurement and the characteristics of beam bending, an innovative method based on local feature point matching and the DIC method for measurement of deformation and force for microcantilevers is proposed. There are many blob feature points in microscopic images of microcantilevers. The gray intensity gradient is usually large and a closed region can always be found in the local area around a blob feature point, similarly to the case of the maximally stable extremal region (MSER) algorithm. ${ }^{(30)}$ The large intensity gradient can improve the anti-noise capability of the DIC method. ${ }^{(31)}$ Moreover, as the distribution of correlation coefficients around the blob feature point is always unimodal, ${ }^{(22,23)}$ it proves to be suitable for convergence of the parameters in iteration-based DIC calculation with the NewtonRaphson method (NRM). Inspired by the ideas mentioned above, the blob feature points are first detected in images before and after deformation using the Laplace-of-Gaussian (LOG) detection algorithm. (32) $^{(2)}$

The subpixel positioning accuracy of LOG feature points is an important issue in the displacement calculation of feature points. At present, there are two methods for sub-pixel positioning of a blob feature point, the difference-of-Gaussian (DOG) method proposed by Lowe ${ }^{(33)}$ and the Hessian-affine method proposed by Mikolajczyk and Schmid. ${ }^{(34)}$ These two methods are based on the quadratic surface fitting of LOG response values calculated at an integer pixel position; the sub-pixel position of the blob point is determined by finding the extreme point of the curved surface. The DOG method is suitable for the rigid and integer transformation, while the Hessian-affine method is very complex and only applicable for small affine transformations. The bending of the cantilever is a kind of nonrigid transformation. To correctly calculate the displacements of feature points in such deformation, a new algorithm is proposed for the sub-pixel positioning of LOG feature points. For each LOG feature point, an affineinvariant local closed region regarded as MSER is extracted and an affine-invariant feature descriptor is constructed. The center of gravity of the local closed region is taken as the center of a LOG feature point. Corresponding points in images before and after deformation are matched according to their affine-invariant descriptors. Compared with the 128-dimensional descriptor used in the scale-invariant feature transform (SIFT) algorithm, the new affine-invariant descriptor has only 9 dimensions and is easily constructed. After feature points are positioned and matched, the DIC method is used to improve the calculation accuracy of feature point displacements. The DIC algorithm using the NRM is performed for matched points whose transformation parameters such as subpixel displacements and strain can be obtained. Finally, by fitting the displacement of matched points with the beam bending formula under the plane stress condition, the force value as well as the position where the loader applies the force are calculated through the least-squares method. The validity of the proposed method is verified through experiments on the actual deformation of a real microcantilever. 
The originalities of the paper are reflected in the following aspects: first, a new positioning method with subpixel resolution is proposed on the basis of the texture information obtained in $\S 2$ and the feature of bending deformation; second, feature points are matched with a new affine moment invariant descriptor, and details for feature point's positioning and matching will be described in $\S 3$. The initialization of the transformation parameters is very important in the DIC application in nonrigid transformation just like the bending deformation. ${ }^{(35,36)}$ The feature point's displacements can be calculated with the positioning and matching information, which can be used for the parameter initialization in the DIC and is described in $\S 4$.

According to the characteristics of bending deformation of the microcantilever, a new bending force calculation model is proposed in $\S 5$, and applied force information can be obtained with the new calculation model. With the new calculation model, the validity of the new feature point's positioning method is verified through the comparison with the positioning method (DOG) ${ }^{(33)}$ used by Lowe and the Hessian-affine method used by Mikolajczyk and Schmid. ${ }^{(34)}$ Using the third-order DIC initialized by the proposed method, more precision displacement information of the feature point, which can be used in the bending force calculation model, can be obtained. Experimental results show that the minimum relative error of the model is within $1.179 \%$.

\section{Image Acquisition of Microcantilever and Image Preprocessing}

A microcantilever with electroplated $\mathrm{Ni}$ is fabricated by micromachining and a bending manipulation device is designed. The microcantilever's dimensions are shown in Fig. 1, where length, width, and thickness are denoted as $l, h$, and $b$, respectively. $L$ represents the actual distance between the point where the force $F$ is applied and the edge of the sample base. The image acquisition system includes a microscope, a 16 bit
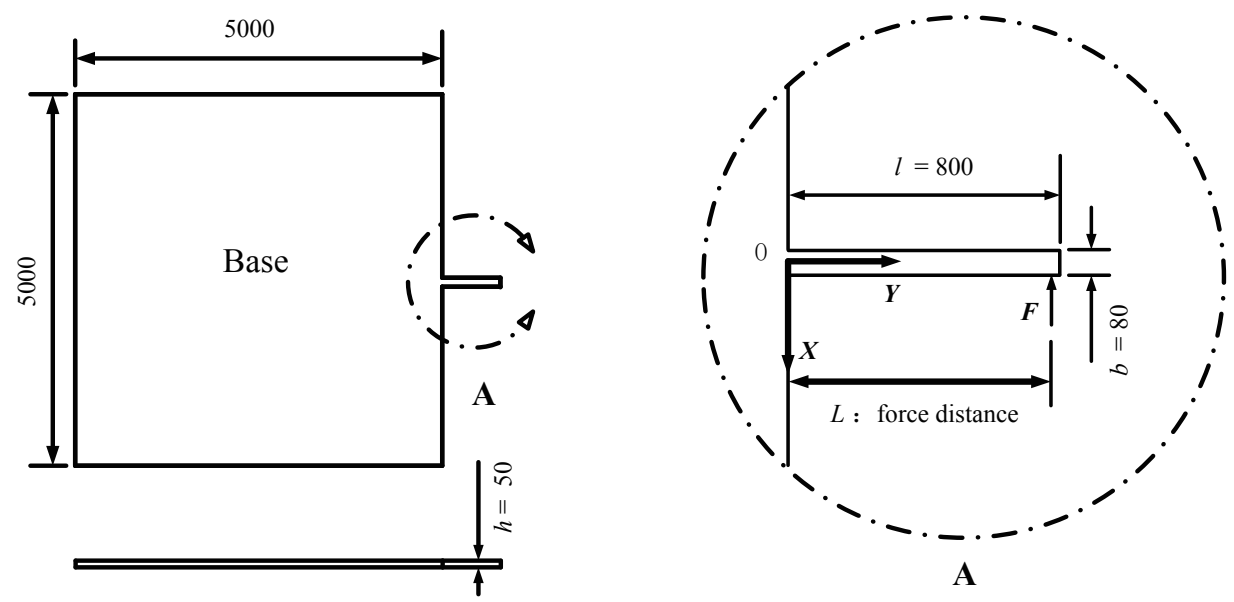

Fig. 1. Dimensions of the microcantilever (unit: $\mu \mathrm{m}$ ). 
charge-coupled diode (CCD) camera, a microdisplacement platform and a computer. The microscope is mounted with the CCD camera that transfers the digital signal to the computer using an IEEE 1394 interface card. A photograph of the image acquisition system is shown in Fig. 2. Images of the microcantilever captured by the CCD camera with a $4 \times$ objective lens before and after force loading are shown in Figs. 3(a) and 3(b), respectively. In Fig. 3, the vertical rod is the cantilever with the loader located on the top left. The loader is mounted on a three-degree-of-freedom micropositioner with $0.1 \mu \mathrm{m}$

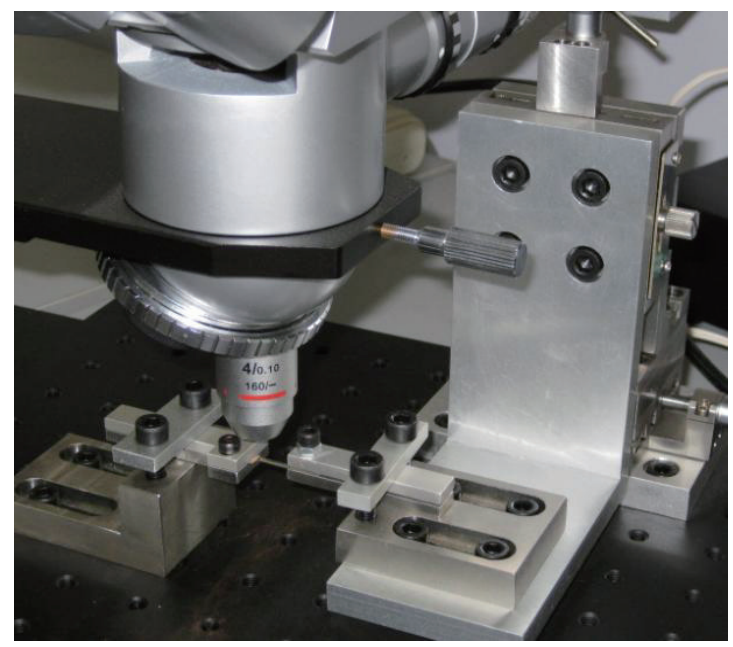

Fig. 2. (Color online) Image acquisition system used in the experiment.

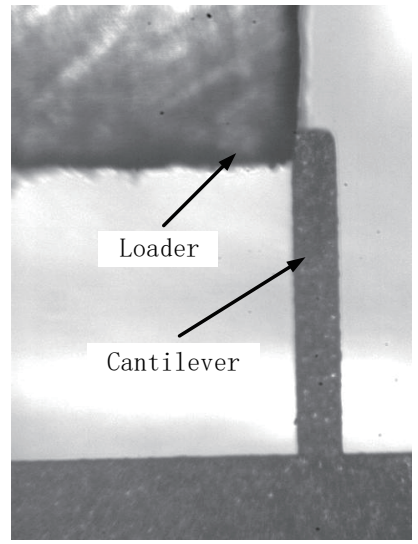

(a)

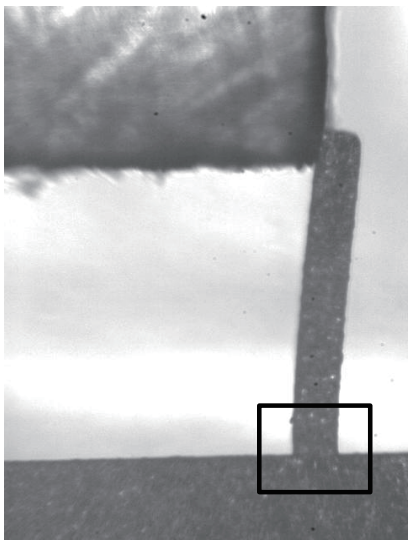

(b)

Fig. 3. Images of the microcantilever before (a) and after (b) force loading. 
positioning resolution along each axis. The straight cantilever will bend once force is applied by the loader. To obtain abundant texture information in the microscopic images, a $10 \times$ objective lens is used to focus the view field of the microscope in the rectangular region shown in Fig. 3(b). To enhance the texture effect, the gray values of images are normalized to $0-1$. Images of the root part of the microcantilever before and after deflection with gray level normalization are shown in Figs. 4(a) and 4(b), respectively. The spatial resolution of the cantilever image is $r=0.5(\mu \mathrm{m} / \mathrm{pixel})$.

\section{Detection and Matching of Feature Points}

\subsection{Feature point detection using the LOG detector}

The LOG detection algorithm is used in Figs. 4(a) and 4(b). The image gray value is denoted by $f(x, y)$. The image is convolved with a Gaussian kernel $h(x, y)$ at the scale $\sigma(\sigma$ $>0)$ and denoted by $I(x, y ; \sigma)$.

$$
\begin{gathered}
I(x, y ; \sigma)=\int f(x-p, y-q) h(p, q ; \sigma) d p d q=f(x, y) \times h(x, y ; \sigma) \\
h(x, y ; \sigma)=\frac{1}{2 \pi \sigma^{2}} e^{\frac{x^{2}+y^{2}}{2 \sigma^{2}}}
\end{gathered}
$$

At the convolution scale $\sigma$, the $(\alpha+\beta)$ th-order derivative of $I(x, y ; \sigma)$ along the $x$ - and $y$-axes is

$$
I_{x^{\alpha} y^{\beta}}(x, y ; \sigma)=\partial_{x^{\alpha} y^{\beta}} I(x, y ; \sigma)=h_{x^{\alpha} y^{\beta}}(x, y ; \sigma) \times f(x, y) .
$$

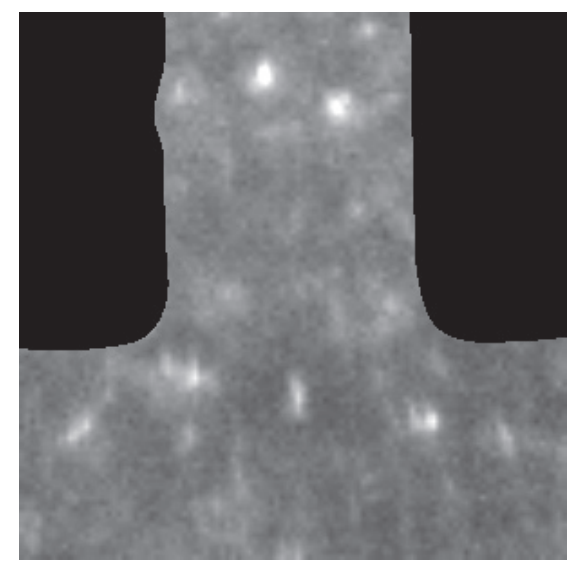

(a)

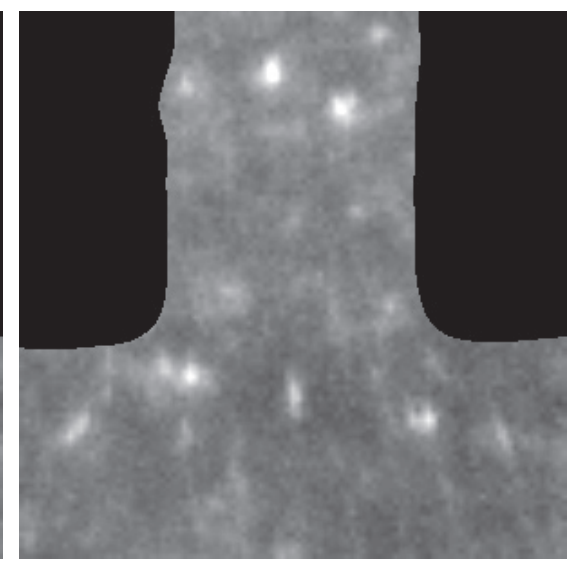

(b)

Fig. 4. Images of the root part of the microcantilever before (a) and after (b) deflection with graylevel normalization. 
The formula for the LOG detector is

$$
\Delta I=\nabla^{2} I=(\nabla \cdot \nabla) I=\sigma^{2}\left(I_{x x}(x, y ; \sigma)+I_{y y}(x, y ; \sigma)\right) .
$$

In the LOG detection algorithm, $\sigma$ ranges from 1 to 30 pixels. Points with $\Delta I>0.03$ are selected as candidates, from which LOG feature points will be detected after nonmaximal suppression (NMS). For each image in Fig. 4, two hundred LOG points with the largest response value are plotted. LOG feature points in Figs. 4(a) and 4(b) are detected and shown in Figs. 5(a) and 5(b), respectively.

\subsection{Construction of feature descriptor}

Each detected LOG feature point $p t$ contains four properties, including response $r(p t)$, scale $s(p t)$, and location $(x(p t), y(p t))$, as denoted by "e+" in Fig. 6. The scale $s(p t)$ equals to the convolution scale $\sigma$ at which the LOG point $p t$ is detected. Six LOG feature points before and after the microcantilever deformation are shown in the first row of Fig. 6, where $\mathrm{a}(1), \mathrm{a}(3)$, and $\mathrm{a}(5)$, and $\mathrm{a}(2), \mathrm{a}(4)$, and $\mathrm{a}(6)$ are the corresponding features before and after the deformation. The location $(x(p t), y(p t))$ of $p t$ is at the integer pixel position calculated by the NMS method. To correctly calculate the displacements of feature points in bending deformation, a new algorithm is proposed for the subpixel positioning of LOG feature points.

Centered at the feature point's location, a local rectangular area is defined with a width that is 6 times the scale of $p t$, as shown in Figs. $6[\mathrm{a}(1)]-6[\mathrm{a}(6)]$. With gray values

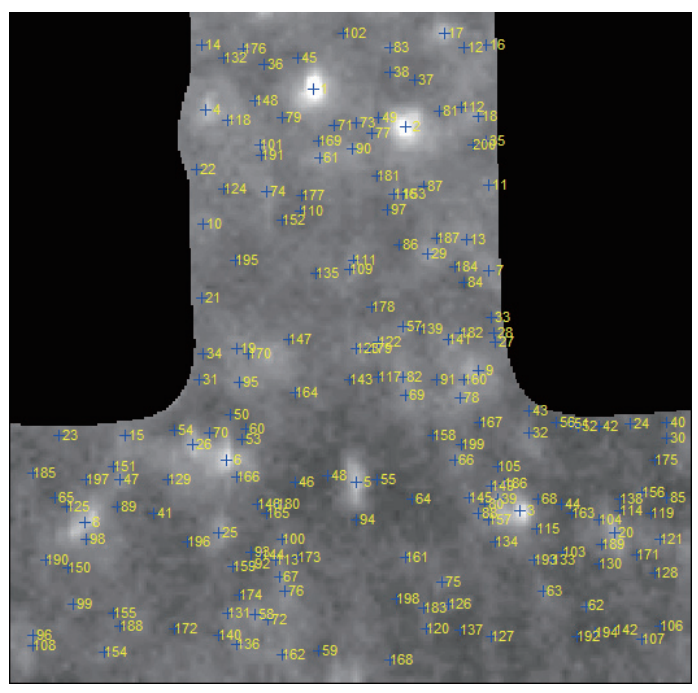

(a)

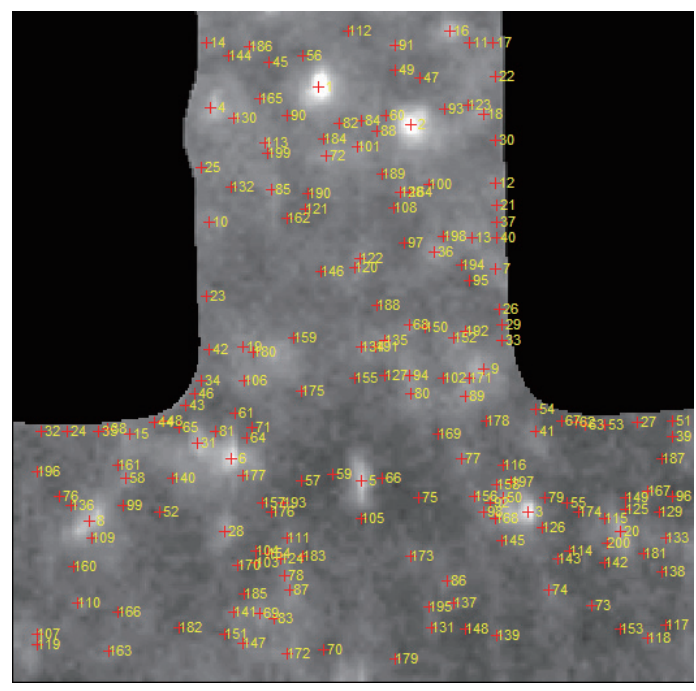

(b)

Fig. 5. (Color online) Results of LOG detector in images before (a) and after (b) deflection. 


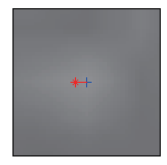

$a(1)$

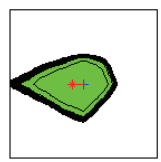

$b(1)$

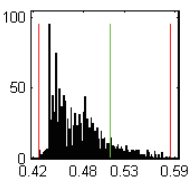

$c(1)$

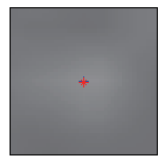

$a(2)$

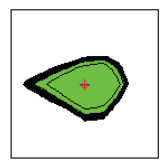

$b(2)$

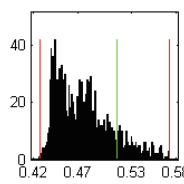

c(2)

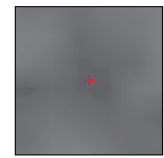

$a(3)$

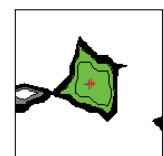

$b(3)$

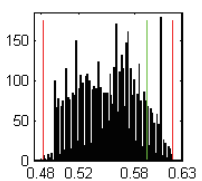

c(3)

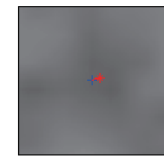

$a(4)$

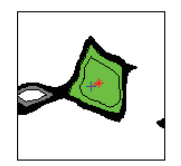

$b(4)$

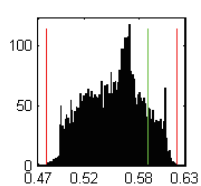

c(4)

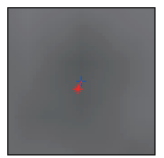

$a(5)$

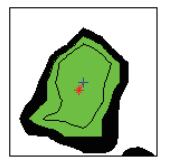

$b(5)$

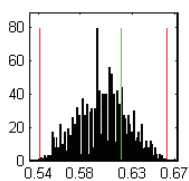

$\mathrm{c}(5)$

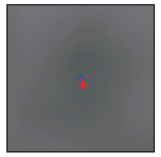

$a(6)$

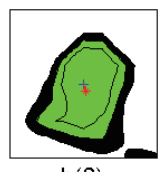

$b(6)$

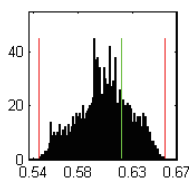

$c(6)$

Fig. 6. (Color online) LOG point and its feature region.

indicated as $f(p t)$, their histograms are displayed in Figs. $6[\mathrm{c}(1)]-6[\mathrm{c}(6)]$. If the feature has a negative LOG response value, then $\operatorname{sign}(p t)$ equals 1 and the feature is indicated as a bright spot (brighter in the center than at the border), as illustrated in Figs. $6[\mathrm{a}(1)]$ and $[\mathrm{a}(2)]$. If the LOG response is positive, then $\operatorname{sign}(\mathrm{pt})$ equals -1 and the feature is indicated as a dark spot, as shown in Figs. $6[\mathrm{a}(3)]$ and $6[\mathrm{a}(5)]$. In order to extract the region in the contours as the inner central region with higher gray values, the dark spot is adjusted in terms of its gray value as $f_{\text {bright }}=1-f_{\text {dark }}$, and then all feature points are adjusted to be bright spots.

The local close region extraction algorithm for each bright feature point is described as follows:

1. By taking the image location $(x(p t), y(p t))$ of a feature point $p$ as the center, and 0.3 times $s(p t)$ as the radius, the average grayscale in this circle is estimated as mean $(f(p t))$, and the threshold $t(p t)$ of the grayscale for the local close region is calculated as $t(p t)=0.7 \times$ mean $(f(p t))$.

2. By using $t(p t)$ as the height, the contour with the same height is extracted from the feature point's neighbors.

3. If the maximum width or height of the contour is 1.5 times larger than $s(p t)$, then $t(p t)$ $=t(p t)+0.005$, and go to step 2, otherwise, go to step 5 .

4. If the maximum width or height of the contour is smaller than 0.3 times $s(p t)$, then $t(p t)=t(p t)-0.005$, and go to step 2, otherwise, go to step 5 .

5. By using the patch inside the contour as a local region of the LOG point $p t(\operatorname{LROL}(p t))$, as illustrated in Figs. $6[\mathrm{~b}(1)]$ and $6[\mathrm{~b}(2)]$, it can be used as a MSER to calculate the feature vector of $p t$.

6. By estimating the center of gravity of the LROL ( $p t)$, the feature point's new location $(c x(p t), c y(p t))$ is used. 
For each detected LOG feature, the corresponding LROL is searched according to the above steps. The center of gravity of LROL is taken as the new center location for the feature (indicated as "**" in the second row of Fig. 6). The threshold for $t(p t)$ is denoted by the vertical green line as shown in Figs. $6[\mathrm{c}(1)]-6[\mathrm{c}(6)]$. All the constants used in the above steps, such as 0.7 used in step $1,1.5$, and 0.005 used in step 3, and 0.3 used in step 4, are determined from the experimental results to obtain a suitable LROL in size and shape for each blob point.

For a LOG point with a LROL, three new properties are added including the feature point's grayscale threshold for the local close region $(t(p t))$ and new location $(c x(p t)$, $c y(p t))$. On the basis of these properties, a descriptor is constructed for this LOG feature point by calculating 6 affine-invariant moments ${ }^{(37)}$ based on the feature's region LROL $(p t)$. Here, $m_{p q}$ denotes the $(p+q)$ th-order geometric moments, and $\mu_{p q}$ denotes the $(p+q)$ th-order central moments:

$$
\begin{gathered}
m_{p q}=\iint_{x, y \in \operatorname{LROL}(p t)} f(x, y) x^{p} y^{q} d x d y \quad p, q=0,1,2,3, \ldots, \\
\left(x_{0}, y_{0}\right)=\left(\frac{m_{10}}{m_{00}}, \frac{m_{01}}{m_{00}}\right), \\
\mu_{p q}=\iint_{x, y \in \operatorname{LROL}(p t)} f(x, y)\left(x-x_{0}\right)^{p}\left(y-y_{0}\right)^{q} d x d y, \\
A M I_{1}=\left(\mu_{20} \mu_{02}-\mu_{11}^{2}\right) / \mu_{00}^{4}, \\
A M I_{2}=\left(-\mu_{30}^{2} \mu_{03}^{2}+\mu_{30} \mu_{21} \mu_{12} \mu_{03}-4 \mu_{30} \mu_{12}^{3}-4 \mu_{21}^{3} \mu_{03}+3 \mu_{21}^{2} \mu_{12}^{2}\right) / \mu_{00}^{10}, \\
A M I_{3}=\left(\mu_{20} \mu_{21} \mu_{03}-\mu_{20} \mu_{12}^{2}-\mu_{11} \mu_{30} \mu_{03}+\mu_{11} \mu_{21} \mu_{12}-\mu_{02} \mu_{21}^{2}\right) / \mu_{00}^{7}, \\
A M I_{4}=\left(\mu_{40} \mu_{04}-4 \mu_{31} \mu_{13}+3 \mu_{22}^{3}\right) / \mu_{00}^{6}, \\
A M I_{6}=\left(\mu_{20}^{2} \mu_{04}-4 \mu_{20} \mu_{11} \mu_{13}+2 \mu_{20} \mu_{02} \mu_{22}+4 \mu_{11}^{2} \mu_{22}-\mu_{11} \mu_{02} \mu_{31}+\mu_{02}^{2} \mu_{40}\right) / \mu_{00}^{7} .
\end{gathered}
$$

With these affine-invariant moments, an affine-invariant feature descriptor is built for each LOG point as

$$
A f f(p t)=\left[\operatorname{sign}(p t) r(p t) t(p t) A M I_{1} A M I_{2} A M I_{3} A M I_{4} A M I_{5} A M I_{6}\right] .
$$




\subsection{Matching of feature points}

If the LOG feature sets with LROLs from the images obtained before and after deformation are $\mathrm{PI}_{1}$ and $\mathrm{PJ}_{1}$, the matching process for a feature point $p_{i} \in \mathrm{PI}_{]}$is described as follows.

1. A new set $\mathrm{PJ}_{2}$ is obtained by excluding points with a different $\operatorname{sign}$ with $\operatorname{sign}\left(p_{i}\right)$ from $\mathrm{PJ}_{1}$.

2. For points in $\mathrm{PJ}_{2}$, the point $p_{j} \in \mathrm{PJ}_{2}$ satisfying the following condition will be selected to obtain a new set $\mathrm{PJ}_{3}$,

$$
\sqrt{\left[c x\left(p_{i}\right)-c x\left(p_{j}\right)\right]^{2}+\left[c y\left(p_{i}\right)-c y\left(p_{j}\right)\right]^{2}} \leq r_{-} \text {threshold }
$$

where $\left(c x\left(p_{i}\right), c y\left(p_{j}\right)\right)$ and $\left(c x\left(p_{j}\right), c y\left(p_{j}\right)\right)$ are the centers of gravity of $\operatorname{LROL}\left(p_{i}\right)$ and $\operatorname{LROL}\left(p_{j}\right)$, respectively. $r_{-}$threshold represents the scope of search for matching, which depends on the actual experiment and is set to 10 since the maximum pixel displacement is no more than 10 pixels in the current experiment.

3. The corresponding point $q_{j} \in \mathrm{PJ}_{3}$ of $p_{\mathrm{i}}$ can be determined by the following condition

$$
d_{\mathrm{aff}}\left(p_{i}, q_{j}\right) \leq R_{\mathrm{aff}},
$$

where $d_{\text {aff }}\left(p_{i}, q_{j}\right)$ is the minimum distance between affine-invariant descriptors for $p_{i}$ and $p_{j}$ defined by

$$
\begin{gathered}
d_{\mathrm{aff}}\left(p_{i}, q_{j}\right)=\min \left\{\operatorname{dis}_{\mathrm{aff}}\left(p_{i}, q_{j}\right), p_{j} \in \mathrm{PJ}_{3}\right\}, \\
d i s_{\mathrm{aff}}\left(p_{i}, p_{j}\right)=\sqrt{\sum_{k=1}^{6}\left(A M I_{k}\left(p_{i}\right)-A M I_{k}\left(p_{j}\right)\right)^{2}}+\lambda \times \operatorname{abs}\left(t\left(p_{i}\right)-t\left(p_{j}\right)\right) .
\end{gathered}
$$

From the experimental results, correctly corresponded feature pairs have the parameters $\lambda$ set to 0.1 and $R_{\text {aff }}$ to 0.015 .

Taking Fig. 4 as an example, matching results for LOG feature points are shown in Fig. 7. The LOG feature points with LROLs and affine-invariant vectors are represented by green patches of various shapes and sizes.

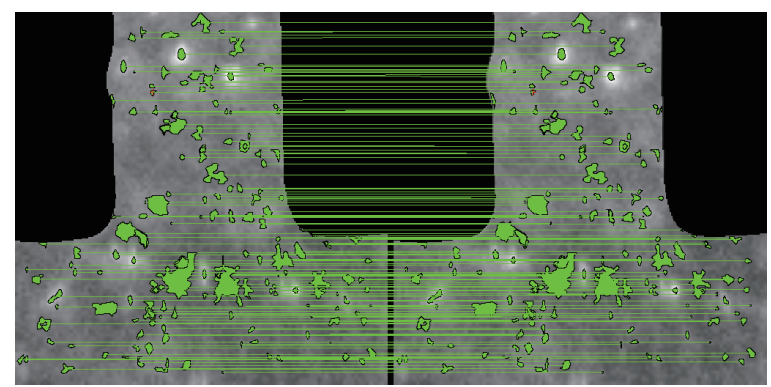

Fig. 7. (Color online) Matching result of LOG feature points in images before and after deformation. 


\section{Displacement Calculation of Feature Point Based on DIC Method}

As mentioned above, the position of a feature point is defined as the center of gravity of its LROL. The displacement of a feature point in the reference image can be calculated from the positions of matched points. For example, if $p_{i}:\left(c x\left(p_{i}\right), c y\left(p_{i}\right)\right)$ in the reference image is matched with $p_{j}:\left(c x\left(p_{j}\right), c y\left(p_{j}\right)\right)$ in the deformed image, the displacement of $p_{i}$ can be calculated as $\Delta x\left(p_{i}\right)=c x\left(p_{j}\right)-c x\left(p_{i}\right), \Delta y\left(p_{i}\right)=c y\left(p_{j}\right)-c y\left(p_{i}\right)$. When the deflection of the cantilever is sufficiently small and in the elastic region, the transformation of LROL for each feature point can be approximated by affine transformation (first-order transformation function) and the corresponding points can be correctly matched by the affine invariant descriptor information. Because the actual transformation of cantilever deflection is not as simple as affine transformation, the center of gravity of LROL in the deformed image is not exactly that of LROL in the reference image; there exists a systematical error for $\Delta x\left(p_{i}\right)$ and $\Delta y\left(p_{i}\right)$ calculated with the center of gravity. To obtain the displacement of feature points with higher resolution, a more accurate model with the transformation function must be considered.

Following the cantilever's bending deflection equation, ${ }^{(38)}$ when a force $F$ is applied at the endpoint and perpendicular to the cantilever, as illustrated in Fig. 1, a point $(x, y)$ on the cantilever has the $x$-axial displacement $\mathrm{u}$ and the $y$-axial displacement $v$ as follows:

$$
\begin{gathered}
u(x, y)=\frac{v F(l-y) x^{2}}{2 E I_{X Z}}+\frac{F(l-y)^{3}}{6 E I_{X Z}}-\frac{F l^{2}(l-y)}{2 E I_{X Z}}+\frac{F l^{3}}{3 E I_{X Z}}, \\
v(x, y)=-\frac{F(l-y)^{2} x}{2 E I_{X Z}}-\frac{v F x^{3}}{6 E I_{X Z}}+\frac{F x^{3}}{6 \mu E I_{X Z}}-\frac{F x^{2}}{2 \mu E I_{X Z}}\left(\frac{b}{2}\right)^{2}+\frac{F l^{2} x}{3 E I_{X Z}}, \\
\mu=\frac{E}{2(1+v)}, \\
I_{X Z}=\frac{h b^{3}}{12},
\end{gathered}
$$

where $E$ is the elastic modulus, $v$ is Poisson's ratio, $\mu$ is Lame's coefficient, and $I_{x z}$ is the moment of inertia for the $X Z$ plate. The $Z$-axis is determined according to the right-hand rule used for coordinates. It can be inferred from eqs. (19) and (20) that the third-order transformation function is required to accurately describe the deformation.

DIC has been widely used for determination of transformation parameters of complex deformation. The reference image obtained before deformation is denoted by $f(x, y)$ and the deformed image is denoted by $g(x, y)$.

From the assumption that the image gray value for the same physical point before and after deformation is constant, we obtain 


$$
f(x, y)=g(x+u, y+v)
$$

where $u(x, y)$ and $v(x, y)$ denote the transformation functions applied to the reference image. As illustrated in Fig. 8, a square patch from the reference image centered at a feature point $\left(c x\left(p_{i}\right), c y\left(p_{i}\right)\right)$ is labeled as a reference subimage, with an edge length equal to $2 \times s\left(p_{i}\right)+1$, where $s\left(p_{i}\right)$ is the scale of the point $p_{i}$.

If the displacement of the point at $\left(c x\left(p_{i}\right), c y\left(p_{i}\right)\right)$ in the reference subimage is set to $(a 1$, $a 2$ ), the displacement of the point $t_{i}$ in the reference subimage can be estimated by thirdorder Taylor expansion as

$$
\begin{gathered}
u\left(x\left(t_{i}\right), y\left(t_{i}\right)\right)=a 1+b 1 \times \Delta x+c 1 \times \Delta y+d 1 \times(\Delta x)^{2}+e 1 \times \Delta x \Delta y+f 1 \times(\Delta y)^{2} \\
+g 1 \times(\Delta x)^{3}+h 1 \times(\Delta x)^{2} \Delta y+i 1 \times \Delta x(\Delta y)^{2}+j 1 \times(\Delta y)^{3}, \\
v\left(x\left(t_{i}\right), y\left(t_{i}\right)\right)=a 2+b 2 \times \Delta x+c 2 \times \Delta y+d 2 \times(\Delta x)^{2}+e 2 \times \Delta x \Delta y+f 2 \times(\Delta y)^{2} \\
+g 2 \times(\Delta x)^{3}+h 2 \times(\Delta x)^{2} \Delta y+i 2 \times \Delta x(\Delta y)^{2}+j 2 \times(\Delta y)^{3} .
\end{gathered}
$$

( $\Delta x, \Delta y$ ) is the offset of $t_{i}$ from the point $p_{i} \cdot p_{i}$ and $t_{i}$ are respectively transformed to $p_{i}{ }^{\prime}$ and $t_{i}{ }^{\prime}$ after deformation. The coefficients of the transformation function are determined by Taylor expansion coefficients as

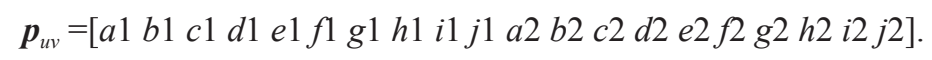

As illustrated in Fig. 8, a new point set is obtained by transforming the points in the reference subimage according to eqs. (24) and (25). A new subimage in the deformed

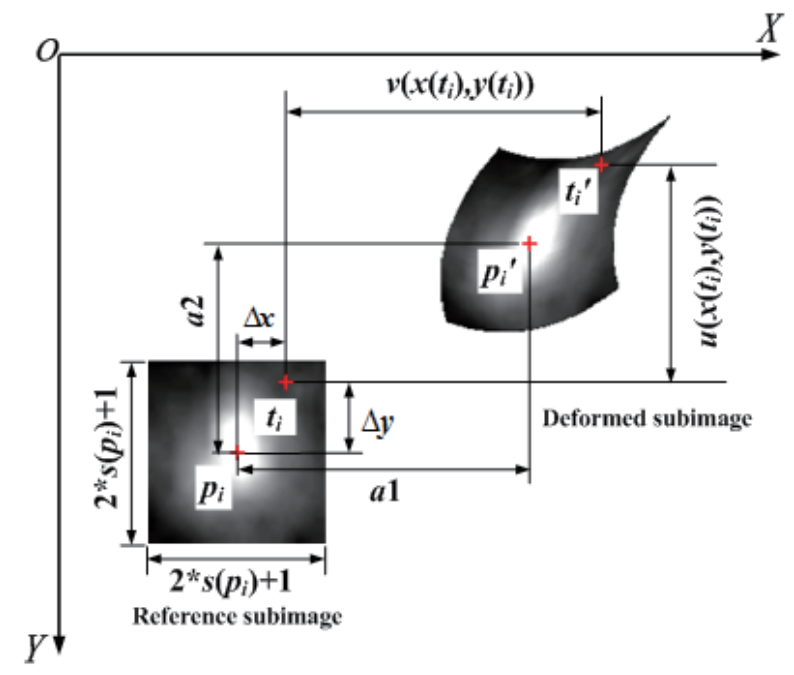

Fig. 8. (Color online) Reference and deformed subimages. 
image is constructed from these new points and is called the deformed subimage with their gray values calculated through bicubic interpolation. According to ref. 25, the correlation between the reference subimage centered at $p_{i}$ and the deformed subimage centered at $p_{i}{ }^{\prime}$ can be estimated by the robust zero-mean normalized sum of squared difference (ZNSSD).

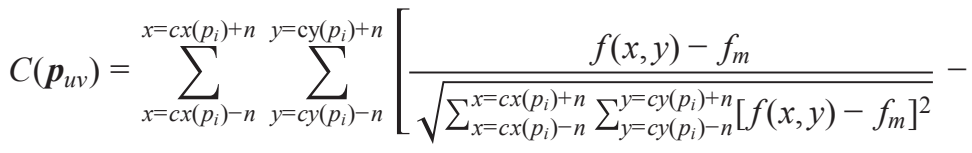

$$
\begin{aligned}
& \left.\frac{g(x+u, y+v)-g_{m}}{\sqrt{\sum_{x=c x\left(p_{i}\right)^{-n}}^{x=c x\left(p_{i}+n\right.} \sum_{y=c y\left(p_{i}\right)-n}^{y=c y\left(p_{i}\right)+n}\left[g(x+u, y+v)-g_{m}\right]^{2}}}\right]^{2} \\
& f_{m}=\frac{1}{(2 n+1)^{2}} \sum_{x=c x\left(p_{i}\right)-n}^{x=c x\left(p_{i}\right)+n} \sum_{y=c y\left(p_{i}\right)-n}^{y=c y\left(p_{i}\right)+n} f(x, y) \\
& g_{m}=\frac{1}{(2 n+1)^{2}} \sum_{x=c x\left(p_{i}\right)-n}^{x=c x\left(p_{i}\right)+n} \sum_{y=c y\left(p_{i}\right)-n}^{y=c y\left(p_{i}\right)+n} g(x+u, y+v)
\end{aligned}
$$

Here, $n=s\left(p_{i}\right)$, and $f_{m}$ and $g_{m}$ are the mean gray values of the reference and deformed subimages, respectively.

Finally, the coefficients of the transformation function can be calculated by iteratively minimizing ZNSSD using the NRM:(25)

$$
\boldsymbol{p}_{u v}^{k+1}=\boldsymbol{p}_{u v}^{k}-\left(\nabla \nabla C\left(\boldsymbol{p}_{u v}^{k}\right)\right)^{-1} \nabla C\left(\boldsymbol{p}_{u v}^{k}\right),
$$

where $\nabla C\left(\boldsymbol{p}_{u v}^{k}\right)$ and $\nabla \nabla C\left(\boldsymbol{p}_{u v}^{k}\right)$ are the first- and second-order derivatives of the correlation function with respect to the coefficients of the transformation function. In the implementation of the NRM, the gray value and its derivative at a subpixel location are obtained by bicubic interpolation.

When deformation is large or there are many transformation parameters to be optimized for the third-order transformation function, the iteration-based DIC method normally requires an accurate initial inference of the deformation to achieve a good convergence result. When a pair of feature points $\left(p_{i}, p_{j}\right)$ is matched from images before and after deformation, in eq. (26), we obtain

$$
\begin{aligned}
& a 1_{\text {initial }}=c x\left(p_{j}\right)-c x\left(p_{i}\right), \\
& a 2_{\text {initial }}=c y\left(p_{j}\right)-c y\left(p_{i}\right),
\end{aligned}
$$

with all other coefficients set to zero to $\left(\boldsymbol{p}_{u v}\right)_{\text {initial }}$. Then, $\left(\boldsymbol{p}_{u v}\right)_{\text {initial }}$ will be used as the initial 
value in the iterative NRM process. The terminal conditions for iterations are

$$
\begin{gathered}
\Delta=p^{k+1}-p^{k}<10^{-6}, \\
p \in\left\{a_{n}, b_{n}, c_{n}, d_{n}, e_{n}, f_{n}, g_{n}, h_{n}, i_{n}, j_{n} ; n=1,2\right\},
\end{gathered}
$$

where $p^{k}$ is the parameter obtained after the $k$ th iteration. After convergence, the displacements of $p_{i}$ in the $x$ - and $y$-directions are

$$
\begin{aligned}
& u\left(x\left(p_{i}\right), y\left(p_{i}\right)\right)=a 1\left(p_{i}\right), \\
& v\left(x\left(p_{i}\right), y\left(p_{i}\right)\right)=a 2\left(p_{i}\right) .
\end{aligned}
$$

\section{Force Measurement Experiments and Discussion}

\subsection{Force measurement based on displacement error function}

The cantilever's bending deflection ${ }^{(38)}$ is described in eqs. (19) and (20). An important issue in the displacement estimation is the determination of the origin of the microcantilever for cantilever deflection. As illustrated in Fig. 9(a), owing to the manufacturing tolerance of the microcantilever, the coordinates of the origin are no longer directly available, as shown in Fig. 1, and should be estimated from the edge information of a real cantilever. In Fig. 9(a), right and left horizontal lines are obtained by fitting the base edge. The initial estimation of $x_{o}$ can be calculated as the mean height of the lines. Similarly, two vertical lines are obtained by fitting the cantilever edges on

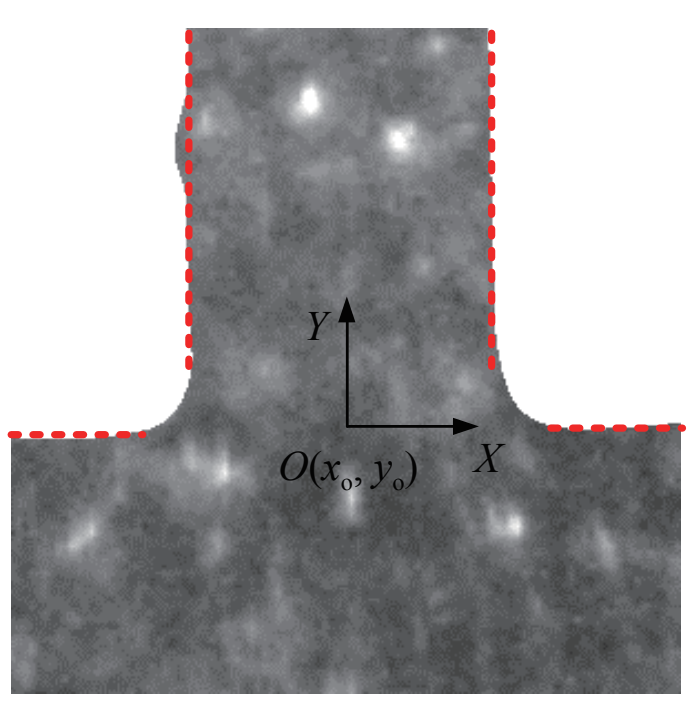

(a)

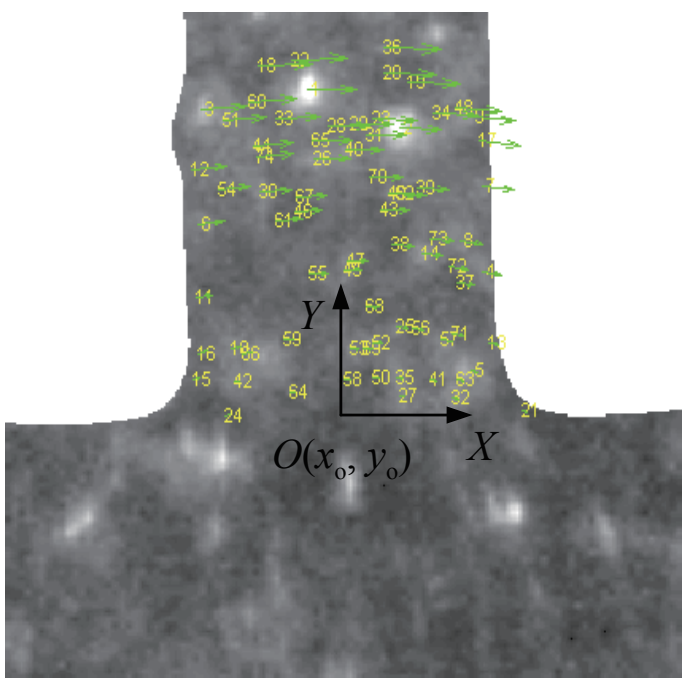

(b)

Fig. 9. (Color online) Deflection coordinates and displacements of points with positive $y$ coordinate: (a) coordinates and (b) displacements. 
the right and left sides, and then $y_{o}$ can be determined as the midpoint of the two vertical lines.

The estimated value of $\left(x_{o}, y_{o}\right)$ is denoted as $\left(x_{\text {ini }}, y_{\text {ini }}\right)$. Positions of the feature points on the cantilever can then be recalculated as $x_{p_{i}}=c x\left(p_{i}\right)-x_{\text {ini }}, y_{p_{i}}=c y\left(p_{i}\right)-y_{\text {ini. }}$. As shown in Fig. 9(b), a point set $P I_{c}$ comprises feature points higher than $y_{\text {ini }}$ :

$$
P I_{c}=\left\{p_{i} ; y_{p_{i}}>y_{\text {ini }}\right\} \text {. }
$$

For every point in $P I_{c}$, theoretical displacements in the $x$ - and $y$-directions can be calculated as

$$
\begin{gathered}
u_{\mathrm{app}}\left(x_{p_{i}}, y_{p_{i}}\right)=\frac{v F\left(L-y_{p_{i}}\right) x_{p_{i}}^{2}}{2 E I_{X Z}}+\frac{F\left(L-y_{p_{i}}\right)^{3}}{6 E I_{X Z}}-\frac{F l^{2}\left(l-y_{p_{i}}\right)}{2 E I_{X Z}}+\frac{F l^{3}}{3 E I_{X Z}}, \\
v_{\mathrm{app}}\left(x_{p_{i}}, y_{p_{i}}\right)=-\frac{F\left(L-y_{p_{i}}\right)^{2} x_{p_{i}}}{2 E I_{X Z}}-\frac{v F x_{p_{i}}^{3}}{6 E I_{X Z}}+\frac{F x_{p_{i}}^{3}}{6 \mu E I_{X Z}}-\frac{F x_{p_{i}}^{2}}{2 \mu E I_{X Z}}\left(\frac{b}{2}\right)^{2}+\frac{F l^{2} x_{p_{i}}}{3 E I_{X Z}} .
\end{gathered}
$$

The force distance $L$ is the projection distance between the point where the force is applied and the coordinates of the origin $\boldsymbol{O}$ along the $y$-axis. The displacements measured with the method proposed in $\S 4$ are shown in Fig. 9(b).

$$
\begin{aligned}
& u_{\mathrm{dic}}\left(x_{p_{i}}, y_{p_{i}}\right)=a 1\left(p_{i}\right) \\
& v_{\mathrm{dic}}\left(x_{p_{i}}, y_{p_{i}}\right)=a 2\left(p_{i}\right)
\end{aligned}
$$

A displacement error function is defined as the sum of square errors of the distance between the theoretical and measured displacements.

$R\left(F, L, x_{\mathrm{o}}, y_{\mathrm{o}}\right)=\sum_{p_{i} \in P I_{c}}\left(\left[u_{\mathrm{dic}}\left(x_{p_{i}}, y_{p_{i}}\right)-u_{\mathrm{app}}\left(x_{p_{i}}, y_{p_{i}}\right)\right]^{2}+\left[v_{\mathrm{dic}}\left(x_{p_{i}}, y_{p_{i}}\right)-v_{\mathrm{app}}\left(x_{p_{i}}, y_{p_{i}}\right)\right]^{2}\right)$

It is a function of the applied force $F$, the force distance $L$ and the coordinates $\left(x_{o}, y_{o}\right)$ of the origin $\boldsymbol{O}$. The parameter vector $\boldsymbol{p}_{f}=\left(F, L, x_{o}, y_{o}\right)$ can be resolved using the NRM as used in DIC mentioned above.

$$
\boldsymbol{p}_{f}^{k+1}=\boldsymbol{p}_{f}^{k}-\left(\nabla \nabla R\left(\boldsymbol{p}_{f}^{k}\right)\right)^{-1} \nabla R\left(\boldsymbol{p}_{f}^{k}\right)
$$

Here, $\nabla R\left(\boldsymbol{p}_{f}^{k}\right)$ and $\nabla \nabla R\left(\boldsymbol{p}_{f}^{k}\right)$ are the first- and second-order derivatives of the error function with respect to the parameter vector $\boldsymbol{p}_{f}=\left(F, L, x_{o}, y_{o}\right)$ at the $k$ th iteration, respectively. The initial value for the iterative process is set to $\boldsymbol{p}_{f}^{1}=\left(0, l, x_{\mathrm{ini}}, y_{\mathrm{ini}}\right)$, where $l$ is the length of the cantilever. When this error function is minimized, the force $F$, the position of the applied force denoted by $L$, and the coordinates of the origin $\left(x_{o}, y_{o}\right)$ can be obtained. 


\subsection{Experimental results and discussion}

As illustrated in Fig. 1, the cantilever is bent by the loader with ten prior-known displacement inputs $(1-10 \mu \mathrm{m})$. The contact point of the loader and the cantilever is near the cantilever tip on the left-hand side. The dimensions of the cantilever are $l=800$ $\mu \mathrm{m}, b=80 \mu \mathrm{m}$, and $h=50 \mu \mathrm{m}$. The cantilever is made of electroplated nickel with its elastic modulus $E=168 \mathrm{GPa}$ calibrated by a microindentation instrument, and Poisson's ratio $v=0.291$. Using the method proposed in this study, the parameter vector $\boldsymbol{p}_{f}=(F$, $\left.L, x_{o}, y_{o}\right)$ is computed for every displacement input. The actual $x$ displacement of the contact point $p_{i}\left(x_{p_{i}}, L\right)$ is denoted as $D_{\text {contact. }}$ According to eq. (38), $D_{\text {contact }}=\frac{F L^{3}}{3 E I_{X Z}}$. The input displacement applied by the loader to the cantilever is denoted as $D_{\text {applied. }}$. The displacement error $\Delta D$ is defined as the discrepancy between $D_{\text {contact }}$ and $D_{\text {applied. }}$. For ten known input displacements $D_{\text {applied, }}$, the applied force $F$, the force distance $L$, the actual $x$ displacement of the contact point $D_{\text {contact }}$ and the percentage of displacement errors $\Delta D$ with respect to the input displacement are shown in Table 1. Overall, the average absolute value of $\Delta D / D_{\text {applied }}$ is about $1.179 \%$.

The applied forces are calculated in two other cases. In one case, the measured displacements used by the displacement error function defined in eq. (42) are obtained directly by the center of gravity of a point's local closed regions. The corresponding results are shown in Table 2. In the other case, the measured displacements of feature points are calculated by the DIC method described in $\S 4$, but the initial values for the coefficients of the transformation function are all set to zero, which means that the matching process described in $\S 3$ is not used. The results in this case are shown in Table 3. On analyzing the data shown in Tables 2 and 3, it can be seen that the mean $\Delta D$ obtained by the proposed method is the smallest. The average absolute values of $\Delta D /$ $D_{\text {applied }}$ are $3.527 \%$ in Table 2 and $7.792 \%$ in Table 3, which are respectively 2.99 and 6.61 times larger than that in Table 1 obtained by the proposed method. In Table 3, when the applied displacements $D_{\text {applied }}$ are 1,2, and $6 \mu \mathrm{m}$, the calculated force distance $L$ is

Table 1

Force measurement results obtained using the DIC method with centroid initialization.

\begin{tabular}{lcccc}
\hline $\begin{array}{l}D_{\text {applied }} \\
(\mu \mathrm{m})\end{array}$ & $\begin{array}{c}F \\
(\mathrm{mN})\end{array}$ & $\begin{array}{c}L \\
(\mu \mathrm{m})\end{array}$ & $\begin{array}{c}D_{\text {contact }} \\
(\mu \mathrm{m})\end{array}$ & $\begin{array}{c}\Delta D / D_{\text {applied }} \\
(\%)\end{array}$ \\
\hline 1 & 2.445 & 764.311 & 1.015 & 1.53 \\
2 & 4.768 & 763.223 & 1.972 & -1.42 \\
3 & 7.376 & 762.732 & 3.044 & 1.47 \\
4 & 9.870 & 761.458 & 4.053 & 1.32 \\
5 & 12.347 & 761.237 & 5.066 & 1.31 \\
6 & 14.864 & 759.822 & 6.064 & 1.07 \\
7 & 17.432 & 758.351 & 7.071 & 1.01 \\
8 & 19.577 & 757.913 & 7.927 & -0.91 \\
9 & 22.421 & 757.832 & 9.076 & 0.84 \\
10 & 25.032 & 756.789 & 10.091 & 0.91 \\
\hline
\end{tabular}


Table 2

Force measurement results obtained using centroid displacement of matched points.

\begin{tabular}{lcccc}
\hline $\begin{array}{l}D_{\text {applied }} \\
(\mu \mathrm{m})\end{array}$ & $\begin{array}{c}F \\
(\mathrm{mN})\end{array}$ & $\begin{array}{c}L \\
(\mu \mathrm{m})\end{array}$ & $\begin{array}{c}D_{\text {contact }} \\
(\mu \mathrm{m})\end{array}$ & $\begin{array}{c}\Delta D / D_{\text {applied }} \\
(\%)\end{array}$ \\
\hline 1 & 2.606 & 757.432 & 1.053 & 5.32 \\
2 & 5.181 & 756.724 & 2.088 & 4.41 \\
3 & 7.185 & 755.576 & 2.882 & -3.92 \\
4 & 10.414 & 755.156 & 4.171 & 4.27 \\
5 & 12.884 & 754.928 & 5.155 & 3.11 \\
6 & 15.598 & 754.762 & 6.238 & 3.96 \\
7 & 18.28 & 751.446 & 7.214 & 3.06 \\
8 & 20.932 & 749.918 & 8.21 & 2.63 \\
9 & 22.595 & 747.821 & 8.789 & -2.35 \\
10 & 26.331 & 747.386 & 10.224 & 2.24 \\
\hline
\end{tabular}

Table 3

Force measurement results obtained using the DIC method without initialization.

\begin{tabular}{lcccc}
\hline $\begin{array}{l}D_{\text {applied }} \\
(\mu \mathrm{m})\end{array}$ & $\begin{array}{c}F \\
(\mathrm{mN})\end{array}$ & $\begin{array}{c}L \\
(\mu \mathrm{m})\end{array}$ & $\begin{array}{c}D_{\text {contact }} \\
(\mu \mathrm{m})\end{array}$ & $\begin{array}{c}\Delta D / D_{\text {applied }} \\
(\%)\end{array}$ \\
\hline 1 & 2.217 & 814.735 & 1.115 & 11.51 \\
2 & 4.222 & 820.183 & 2.166 & 8.32 \\
3 & 5.95 & 789.272 & 2.721 & -9.31 \\
4 & 9.388 & 787.299 & 4.261 & 6.52 \\
5 & 11.601 & 791.850 & 5.357 & 7.14 \\
6 & 12.928 & 811.413 & 6.424 & 7.06 \\
7 & 16.205 & 791.372 & 7.470 & 6.71 \\
8 & 18.456 & 797.847 & 8.718 & 8.97 \\
9 & 18.098 & 795.215 & 8.465 & -5.95 \\
10 & 22.918 & 793.343 & 10.643 & 6.43 \\
\hline
\end{tabular}

incorrect, as it is larger than the length of the cantilever, which is $800 \mu \mathrm{m}$. The incorrect force distance $L$ and the largest mean displacement error all validate the necessity of the appropriate initialization of the coefficients of the transformation function used in DIC. The larger mean displacement error in Tables 2 and 3 than in Table 1 shows that the initialization of the coefficients of the transformation function by the matching process described in $\S 3$ is effective.

For comparison with other methods, the displacements of feature points are calculated by different subpixel positioning methods, which are DOG and DOG+DIC (the parameters of DIC are initialized by DOG), and Hessian-affine and Hessian-affine+DIC (the parameters of DIC are initialized by Hessian-affine) methods. Force measurement results obtained using these methods are shown in Tables 4 and 5, respectively. For simplicity, only the force distance $L$ and the percentage of displacement errors $\Delta D$ with 
Table 4

Force measurement results obtained using the DOG and DIC methods.

\begin{tabular}{lcccc}
\hline \multirow{2}{*}{$\begin{array}{l}D_{\text {applied }} \\
(\mu \mathrm{m})\end{array}$} & $\begin{array}{c}c \\
\text { DOG }\end{array}$ & $\begin{array}{c}\Delta D / D_{\text {applied }} \\
(\mu \mathrm{m})\end{array}$ & $\begin{array}{c}L \\
(\mu \mathrm{m})\end{array}$ & $\begin{array}{c}\Delta D / D_{\text {applied }} \\
(\%)\end{array}$ \\
\hline 1 & 978.519 & 258.78 & 1214.557 & 47.18 \\
2 & 1032.897 & 186.27 & 904.237 & 35.24 \\
3 & 1264.812 & 313.15 & 856.436 & 27.15 \\
4 & 1173.214 & 292.47 & 891.134 & 22.71 \\
5 & 1654.739 & 211.92 & 823.401 & -19.34 \\
6 & 1968.414 & 222.37 & 782.268 & 14.28 \\
7 & 2151.113 & 339.33 & 792.719 & 15.59 \\
8 & 1349.247 & 349.45 & 774.391 & 13.37 \\
9 & 1977.354 & 221.35 & 792.304 & 15.27 \\
10 & 2169.521 & 231.32 & 782.491 & 14.81 \\
\hline
\end{tabular}

Table 5

Force measurement results obtained using the Hessian-affine and DIC methods.

\begin{tabular}{lcccc}
\hline \multirow{2}{*}{$\begin{array}{l}\text { applied } \\
(\mu \mathrm{m})\end{array}$} & \multicolumn{2}{c}{ Hessian-affine } & \multicolumn{2}{c}{ Hessian-affine+DIC } \\
\cline { 2 - 5 } & \multicolumn{1}{c}{$\begin{array}{c}\Delta \mathrm{m}) \\
\text { ( }) D_{\text {applied }}\end{array}$} & $\begin{array}{c}L \\
(\%)\end{array}$ & $\begin{array}{c}\Delta D / D_{\text {applied }} \\
(\mu \mathrm{m})\end{array}$ \\
\hline 1 & 1421.443 & 97.41 & 913.435 & 32.23 \\
2 & 1634.453 & 111.21 & 871.225 & 28.11 \\
3 & 1365.221 & 113.34 & 768.114 & -22.57 \\
4 & 997.245 & 92.44 & 782.125 & 13.13 \\
5 & 1721.116 & 121.82 & 784.526 & 17.26 \\
6 & 1435.232 & 143.37 & 768.546 & 15.31 \\
7 & 1462.764 & 129.87 & 780.016 & 13.44 \\
8 & 1554.347 & 99.97 & 788.269 & 12.89 \\
9 & 1674.216 & -41.13 & 755.784 & -25.57 \\
10 & 1548.213 & 101.27 & 785.207 & 16.02 \\
\hline
\end{tabular}

respect to the input displacement are shown. Through the data shown in Tables 4 and 5 , it is obvious that force measurement results are incorrect when the feature point's displacements are calculated by the DOG or Hessian-affine method, because the force distance $L$ values are larger than $800 \mu \mathrm{m}$. When combined with the DIC method, the calculation error for the force measurement results decreases apparently, although there still exists some incorrect calculation for some displacement inputs when $L$ is larger than $800 \mu \mathrm{m}$. The average absolute values of $\Delta D / D_{\text {applied }}$ are 22.494 and $19.653 \%$ for the DOG+DIC and Hessian-affine+DIC methods, respectively, which are significantly larger than those in Tables 1 and 3. The data shown in Tables 4 and 5 verify the effectiveness of the proposed subpixel positioning method for the feature point and the necessity of appropriate initialization for the DIC method. 
The data shown in Table 1 verify the effectiveness of the DIC method used for the deformation and force measurement of the microcantilever. Another thing should be noticed is the texture property of the microcantilever surface, which determines directly the gray value shown in the microscopic image of microcantilever. To evaluate the effect of the texture property of the image on the DIC calculation, Crammond et al. ${ }^{(31)}$ summarized many algorithms to quantify the speckle pattern quality used for the DIC method, such as mean intensity gradient, mean subset fluctuation and subset entropy. These algorithms all show the significant influence of the intensity gradient of the image on controlling the noise resistance of the DIC method. A simple but efficient way is to quantify the significance of the intensity gradient by calculating the variance of the gray value. The noise resistance of the image can be calculated using the signal-to-noise ratio $(\mathrm{SNR}), \mathrm{SNR}=10 \log _{10} \frac{\sigma_{\text {signal }}}{\sigma_{\text {noise }}}$, where $\sigma_{\text {signal }}$ is the variance of the signal (gray value of the image) and $\sigma_{\text {noise }}$ is the variance of noise. The noise resistance of the local square region around the LOG feature point can be calculated using the SNR value. Taking the local regions shown in Figs. 6[a(1)] to $6[\mathrm{a}(6)]$ as an example, the side length of the square region around a LOG point is 6 times the feature scale of the corresponding point. When $\sigma_{\text {noise }}$ is set to 2 (with the gray value of the image between 1 and 255), the maximum, minimum, and mean SNR values of square regions of LOG points shown in Fig. 9(b) are $35.211,24.117$, and 28.725, respectively. The side length of these local square regions in Fig. 9(b) is in the range of 15 to 25 pixels. For comparison, the image of the microcantilever is scanned by a square region with a side length equal to 20 pixels and the SNR value of the corresponding region covered by the scanning square is calculated (with $\sigma_{\text {noise }}$ set to 2). Calculation results show that the maximum, minimum, and mean SNR values are $32.478,2.157$, and 11.295 , respectively. It can be seen that the local regions around LOG feature points have larger SNR values than the normal region, which means that a larger intensity gradient will appear in these regions. Thus, the SNR calculation results of local regions show the advantage of performing DIC calculation around LOG points. This also means that enough texture and gradient information is needed for a surface if its deformation is measured by the DIC method.

\section{Conclusion}

An optical method based on local feature point matching and the DIC method for deformation and force measurement of a microcantilever is proposed. LOG feature points with local extreme response values are detected in the scale space in images obtained before and after deformation. LOG feature points are selected owing to their high intensity gradient in the surrounding region and the unimodal distribution of the correlation coefficient, both of which are good for convergence of parameters used in DIC calculation with the NRM. Affine-invariant feature descriptors are constructed and used for the matching of feature points. From the center of gravity of the feature point's closed region and matching information, the preliminary subpixel displacement of matched points can be estimated and used for the initial value in the DIC calculation with a third-order transformation function. The deformation of the microcantilever can 
be calculated by fitting the displacement of matched points with the beam's bending deflection formula under the plane stress condition. The force values as well as the position where the loader come in contact with the cantilever are calculated through the least-squares method. The coordinates of the origin for cantilever deflection are considered to reduce the error caused by the micromachine tolerance. The prior-known applied displacement is compared with the calculated displacement of the contact point. The relative displacement error of $1.179 \%$ validates the applicability of the proposed method in actual deformation experiments of microcantilevers.

\section{Acknowledgements}

This work is supported by National Natural Science Foundation of China (Grant Numbers 51375303 and 50875169).

\section{References}

1 X. D Li, H. M. Xie and Y. L. Kang: Acta Mech. Solida Sin. 23 (2010) 498.

D. S. Gianola and C. Eberl: JOM-US 61 (2009) 24.

3 Y. Sun, S. N. Fry and D. P. Potasek: J. Microelectromech. Syst. 14 (2005) 4.

4 Y. Sun, K. T. Wan and K. P. Roberts: IEEE Trans. Nanobiosci. 2 (2003) 279.

5 Y. T. Shen, N. Xi, U. C. Wejinya and W. J. Li: Proceedings of IEEE International Conference on Intelligent Robots and Systems, ed. N. M. Amato (IEEE, Sendai International Center, Japan, 2004) p. 3363.

6 T. C. Duc, J. F. Creemer and P. M. Sarro: IEEE Sens. J. 7 (2007) 96.

7 H. K. Chu, J. K. Mills and W. L. Cleghorn: IEEE International Conference on Advanced Intelligent Mechatronics, eds. H. Hashimoto and K. M. Lee (IEEE, Xi' an Jiaotong University, Xi'an, 2008) p. 797.

8 H. J. Butt, B. Cappella and M. Kappl: Surf. Sci. Rep. 59 (2005) 1.

9 J. Nader and L. Karthik: Mechatronics 14 (2004) 907.

10 J. E. Sader, J. A. Sanelli and B. D. Adamson: Rev. Sci. Instrum. 83 (2012) 103705.

11 R. S. Gates, M. G. Reitsma, J. A. Kramar and J. R. Pratt: J. Res. Natl. Inst. Stand. Technol. 116 (2011) 703.

12 S. H. Kim and J. G. Boyd: Meas. Sci. Technol. 17 (2006) 2343.

13 Y. Zhou, B. J. Nelson and B. Vikramaditya: J. Intell. Robot. Syst. 28 (2000) 259.

14 M. A. Greminger and B. J. Nelson: IEEE Trans. Pattern Anal. Mach. Intell. 26 (2004) 290.

15 Y. H. Anis, J. K. Mills and W. L. Cleghorn: J. Micromech. Microeng. 16 (2006) 1639.

16 F. Amiot, F. Hild and J. P. Roger: Int. J. Solids Struct. 44 (2007) 2863.

17 X. D. Li, Y. Yang and C. Wei: Meas. Sci. Technol. 15 (2004) 75.

18 W. Hu, S. G. Wang, C. Hu, H. T. Liu and J. Q. Mo: Proc. Inst. Mech. Eng. Part C-J. Eng. Mech. Eng. Sci. 226 (2012) 2589.

19 W. Hu, H. T. Liu, C. Hu, S. G. Wang, D. Chen, J. Q. Mo and Q. H. Liang: Measurement 46 (2013) 4293.

20 A. N. Reddy, N. Maheshwari, D. K. Sahu and G. K. Ananthasuresh: IEEE Trans. Robot. 26 (2010) 867.

21 X. D. Li, C. Wei and Y. Yang: Opt. Lasers Eng. 43 (2005) 869.

22 Z. W. Liu, H. M. Xie and D. N. Fang: Microelectron. Reliab. 47 (2007) 2226. 
23 Y. L. Kang, H. W. Wang and K. H. Laermann: Proceedings of the 2004 SEM X International Congress and Exposition on Experimental and Applied Mechanics (SEM, Costa Mesa, California, 2004).

24 W. N. Sharpe, J. Pulskamp and D. S. Gianola: Exp. Mech. 47 (2007) 649.

25 B. Pan, Q. Kemao, H. M. Xie and A. Asundi: Meas. Sci. Technol. 20 (2009) 062001.

26 B. Pan, H. M. Xie, B. Q. Xu and F. L. Da: Meas. Sci. Technol. 17 (2006) 1615.

27 D. S. Zhang, M. Luo and D. Arola: Opt. Eng. 45 (2006) 033605.

28 C. Eberl, D. S. Gianola and K. J. Hemker: Exp. Mech. 50 (2010) 85.

29 F. Hild and S. Roux: Strain. 42 (2006) 69.

30 K. Mikolajczyk, T. Tuytelaars and C. Schmid: Int. J. Comput. Vis. 65(2005) 43.

31 G. Crammond, S. W. Boyd and J. M. Dulieu-Barton: Opt. Lasers. Eng. 51 (2013) 1368.

32 K. Mikolajczyk and C. Schmid: IEEE Trans. Pattern Anal. Mach. Intell. 27 (2005) 1615.

33 D. G. Lowe: Int. J. Comput. Vis. 60 (2004) 91.

34 K. Mikolajczyk and C. Schmid: Int. J. Comput. Vis. 60 (2004) 63.

35 Y. H. Zhou and Y. Q. Chen: Opt. Lasers. Eng. 51 (2013) 213.

36 Z. Wang, M. Vo, H. Kier and T. Pan: Strain. 50 (2014) 28.

37 T. Suk and J. Flusser: Pattern Recognit. 44 (2011) 2047.

38 W. M. Lai, D. Rubin and E. Krempl: Introduction to Continuum Mechanics (Elsevier, Burlington, 2009) p. 257. 